\title{
Fast liquid chromatography-electrochemistry-mass spectrometry of ferrocenecarboxylic acid esters
}

\author{
Georg Diehl, Uwe Karst* \\ University of Twente, Department of Chemical Analysis and MESA ${ }^{+}$Research Institute, P.O. Box 217, 7500 AE Enschede, \\ The Netherlands
}

\begin{abstract}
Rapid liquid chromatographic separations of ferrocenecarboxylic esters of various alcohols and phenols have been achieved on reversed-phase columns of $20 \mathrm{~mm}$ length. After separation, the ferrocene derivatives are oxidized electrochemically under formation of the charged ferrocinium species, which are easily detected by mass spectrometry using an atmospheric pressure chemical ionization source operated in the heated nebulizer mode. While a series of nine phenol derivatives was separated within less than $1.5 \mathrm{~min}$, six alcohol derivatives eluted within 1 min. Limits of detection using a single quadrupole mass analyzer ranged from 60 to $190 \mathrm{nmol} / 1$. Additional work was directed on the use of a graphite in-line filter instead of a silica-based reversed-phase column to achieve the separation.
\end{abstract}

(C) 2002 Elsevier Science B.V. All rights reserved.

Keywords: Electrochemistry; Mass spectrometry; Alcohols; Phenols; Ferrocenecarboxylic acid esters

\section{Introduction}

The hyphenation of liquid chromatography and mass spectrometry (LC-MS) has become a widely used technique both in research and routine analysis [1]. The most commonly employed ionization methods are electrospray (ES) ionization [2] and atmospheric pressure chemical ionization (APCI). ES and APCI are suitable for analytes of medium to strong polarity, but often fail in the ionization of compounds that are not easily (de)protonated [3]. To overcome this poor ionization, a few groups have coupled electrochemical techniques to various ionization interfaces in order to use electrochemical

\footnotetext{
*Corresponding author. Tel.: +31-53-489-2983; fax: +31-53489-4645.

E-mail address: u.karst@ct.utwente.nl (U. Karst).
}

conversion of the analytes for better mass spectrometric performance. These approaches have been reviewed in [4,5]. A recent approach has used the on-line coupling of LC-MS with a "coulometric" flow cell for the on-line oxidation of several ferrocene-labeled alcohols and phenols to its corresponding ferrocinium radical cations that were analyzed by mass spectrometry in a single quadrupole mass analyzer [6].

In recent years, the evolution of combinatorial chemistry has posed great demands on the development of analytical methods suitable for high sample throughput. One possible approach is the reduction of the chromatographic run time, as it has been realized by the application of various technologies [7-12]: gigaporous (perfusive, pore diameter greater than one hundreth of the particle diameter) $[7,8]$ or micropellicular $[9,10]$ stationary phases, elevated 
temperature [10] and short (1 to $2 \mathrm{~cm})$ columns [13-15] have been reported. The present study shows that fast LC analyses are possible with LCelectrochemistry-MS, when using guard columns.

\section{Experimental}

\subsection{Chemicals}

Ammonium formate was purchased from Aldrich (Steinheim, Germany), formic acid was obtained from Fluka (Buchs, Switzerland) in the highest purity available. Acetonitrile (LiChroSolv gradient grade) was obtained from Merck (Darmstadt, Germany).

\subsection{Instrumentation}

\subsubsection{Electrochemical instrumentation}

The electrochemical system from ESA (Chelmsford, MA, USA) which was used for on-line LC-electrochemistry-MS consisted of a GuardStat potentiostat and a Model 5021 conditioning cell. The conditioning cell contains a porous glassy carbon "coulometric" working electrode, a Pd counter electrode, and a $\mathrm{Pd} / \mathrm{H}_{2}$ reference electrode. The electrochemical conditioning cell is characterized by a low void volume and a large surface area of the porous glassy carbon working electrode. These electrode properties allow quantitative oxidation or reduction reactions. The term "coulometric" is used by the manufacturer to indicate the quantitative electrochemical conversion of the analyte. The electrochemically correct term for the cell would rather be an amperometric cell with quantitative conversion because no measurement of charge is carried out. Therefore, the term "coulometric" will be used in this work in quotes to emphasize that there is no coulometry involved in the classical meaning of this term. The potential used in this study was $0.7 \mathrm{~V}$ vs. $\mathrm{Pd} / \mathrm{H}_{2}[6]$.

\subsection{2. $L C-M S$ instrumentation}

The LC-MS system from Shimadzu (Duisburg, Germany) consisted of a SCL-10Avp controller unit, a DGU-14A degasser, two LC-10ADvp pumps, an
SUS mixing chamber $(0.5 \mathrm{ml})$ for standard LC separations, or a low-void-volume mixing tee (Upchurch, Oak Harbor, WA, USA) for the fast LC separations, an SIL-10A autosampler, a QP8000 single quadrupole mass spectrometer with APCI probe and Class 8000 Version 1.20 software.

\subsection{LC-electrochemistry-MS setup}

For the on-line integration of the potentiostat controlled electrochemical cell in the existing LCMS system, the cell was inserted between the LC column and the mass spectrometer interface. The connection between flow cell and interface was kept as short as possible, to minimize peak broadening and loss of ions during transport. To prevent electrical connection between interface and "coulometric" cell via the eluent, appropriate ground connection had to be assured as discussed by Zhou and van Berkel [16]. This was accomplished by replacing part of the polyether ether ketone (PEEK) connection between flow cell and MS system with a stainless steel capillary that was in electrical contact with the grounded part of the mass spectrometer. To protect the porous working electrode from particles, PEEK in-line filters (ESA) were inserted between column and electrochemical cell. The schematic setup of the system used for fast separations is shown in Fig. 1.

For rapid LC-electrochemistry-MS with graphite filter elements as stationary phases, the guard column was removed from the setup and the PEEK in-line filter element was replaced by a graphite in-line filter element.

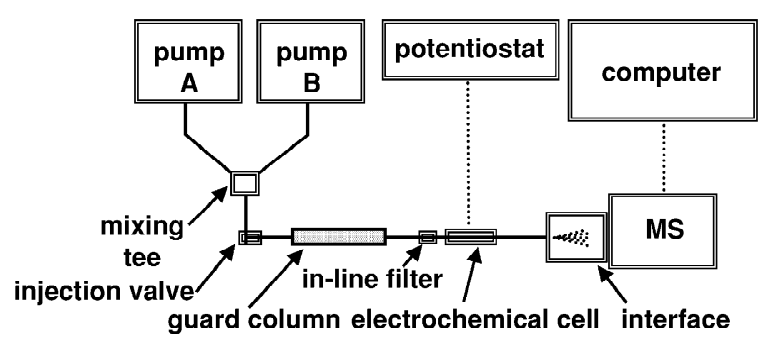

Fig. 1. Schematic setup of the LC-electrochemistry-MS system employed for fast separations over guard columns. 
2.4. Synthesis of ferrocenecarboxylic acid esters (FCEs)

The derivatives were synthesized according to a slightly modified procedure of Rolfes and Andersson $[17,18]$ as described in Ref. [6]. Several alkylphenyl FCE standards were kindly provided by F. Wasinski, J. Rolfes and J.T. Andersson from the University of Münster (Münster, Germany).

\subsection{LC conditions}

Separations with standard time scales were carried out using Discovery $\mathrm{C}_{18}$ columns (Supelco, Deisenhofen, Germany) equipped with guard columns of the same material with the following dimensions: $5 \mu \mathrm{m}$ particle size, $100 \AA$ pore size, $3.0 \mathrm{~mm}$ I.D., 20 $\mathrm{mm}$ length (guard column) and $150 \mathrm{~mm}$ length

Table 1

Profile of gradients: A (injection volume $10 \mu \mathrm{l}$, flow-rate 0.6 $\mathrm{ml} / \mathrm{min}$ ); B (injection volume $5 \mu \mathrm{l}$, flow-rate $1.25 \mathrm{ml} / \mathrm{min}$ ); C (injection volume $5 \mu \mathrm{l}$, flow-rate $1.25 \mathrm{ml} / \mathrm{min}$ ) and $\mathrm{D}$ (injection volume $2 \mu \mathrm{l}$, flow-rate $0.6 \mathrm{ml} / \mathrm{min}$ )

\begin{tabular}{|c|c|c|}
\hline Gradient & $t(\min )$ & $\mathrm{CH}_{3} \mathrm{CN}(\%)$ \\
\hline \multirow[t]{7}{*}{ A } & 0.01 & 60 \\
\hline & 3.00 & 60 \\
\hline & 8.00 & 90 \\
\hline & 18.00 & 90 \\
\hline & 20.00 & 60 \\
\hline & 23.00 & 60 \\
\hline & 23.50 & Stop \\
\hline \multirow[t]{5}{*}{ B } & 0.01 & 50 \\
\hline & 0.90 & 90 \\
\hline & 1.35 & 90 \\
\hline & 1.45 & 50 \\
\hline & 1.50 & Stop \\
\hline \multirow[t]{5}{*}{$\mathrm{C}$} & 0.01 & 40 \\
\hline & 0.80 & 95 \\
\hline & 1.00 & 95 \\
\hline & 1.20 & 40 \\
\hline & 1.25 & Stop \\
\hline \multirow[t]{6}{*}{$\mathrm{D}$} & 0.03 & 20 \\
\hline & 0.20 & 20 \\
\hline & 2.20 & 90 \\
\hline & 3.00 & 90 \\
\hline & 3.50 & 20 \\
\hline & 4.00 & Stop \\
\hline
\end{tabular}

(analytical column). Fast separations were performed using only the $20 \mathrm{~mm}$ length guard columns. Eluent A of the mobile phase was a solution of $250 \mathrm{mg}$ ammonium formate and $0.6 \mathrm{ml}$ formic acid in 11 deionized water $(\mathrm{pH} \approx 3)$. Eluent $\mathrm{B}$ was acetonitrile. Different gradients $(\mathrm{A}-\mathrm{C})$ with the profiles presented in Table 1 were used.

For rapid LC-electrochemistry-MS using graphite filter elements as stationary phases, the separations were performed without the guard column. Gradient $\mathrm{D}$ with the profile provided in Table 1 was used.

\subsection{MS conditions}

The APCI interface was used as heated nebulizer only, without discharge ionization [6], with the following parameters: nebulizer gas flow $2.51 / \mathrm{min}$, APCI temperature $375^{\circ} \mathrm{C}$, APCI probe voltage 0.10 $\mathrm{kV}$, CDL temperature $300^{\circ} \mathrm{C}, \mathrm{CDL}$ voltage $-35 \mathrm{~V}$, deflector voltages $+35 \mathrm{~V}$, sampling rate $10 \mathrm{~Hz}$ for fast separations and $2 \mathrm{~Hz}$ for standard separations.

\section{Results and discussion}

\subsection{Conventional LC-electrochemistry-MS of phenol derivatives}

Before fast LC separations with guard columns were performed, LC-electrochemistry-APCI $(+)$ MS chromatograms of standard time scales were recorded in order to be able to compare the analysis time of these runs with fast LC runs that are described later in this work. A $10 \mu M$ standard mixture containing nine different alkylphenyl ferrocenecarboxylic acid esters was separated on a standard analytical column of $150 \mathrm{~mm}$ length. The separation employing gradient A took place in less than 19 min (Fig. 2). All alkylphenol derivatives were baseline separated, oxidized electrochemically in the "coulometric" flow cell and subsequently detected by MS (Fig. 3). The APCI interface was used as a heated nebulizer only (without discharge), because the radical cations provided by oxidation in solution were efficiently transferred to the gas phase at low probe voltage. Therefore, the set-up could also be referred to as a thermospray interface. In Fig. 4, 


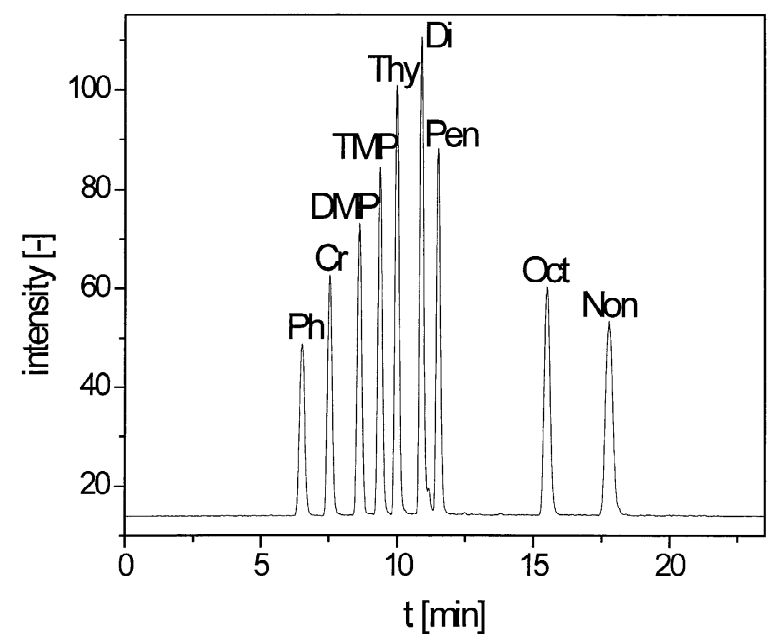

Fig. 2. Conventional LC-electrochemistry-APCI(+)-MS TIC chromatogram, recorded in the SIM mode $(\mathrm{m} / z=306.0,320.1$, 334.1, 348.1, 362.1, 376.1, 390.1, 418.2, 432.2); $\mathrm{Ph}=$ phenyl FCE, $\mathrm{Cr}=2$-cresol $\mathrm{FCE}, \mathrm{DMP}=2,5$-dimethylphenyl $\mathrm{FCE}, \mathrm{TMP}=2,3,6$ trimethylphenyl FCE, Thy=thymol FCE, Di=2,6-diisopropylphenyl FCE, Pen=4- $n$-pentylphenyl FCE, Oct=4- $n$-octylphenyl FCE, Non=4-n-nonylphenyl FCE.

the APCI $(+)$ mass spectrum of a $10 \mu \mathrm{mol} / 1$ solution of 2-cresol FCE is presented. It is obvious that the base peak is the radical cation of the analyte. The $[\mathrm{M}+1]^{+}$peak can be mainly explained by the natural abundance of the ${ }^{13} \mathrm{C}$ isotope in the analyte, and only to a small fraction by protonation of the 2-cresol FCE.

\subsection{Fast LC-electrochemistry-MS of phenol derivatives}

To demonstrate that LC-electrochemistry-AP$\mathrm{CI}(+)$-MS can also be performed with fast LC separations, the same $10 \mu M$ mixture of nine differ-

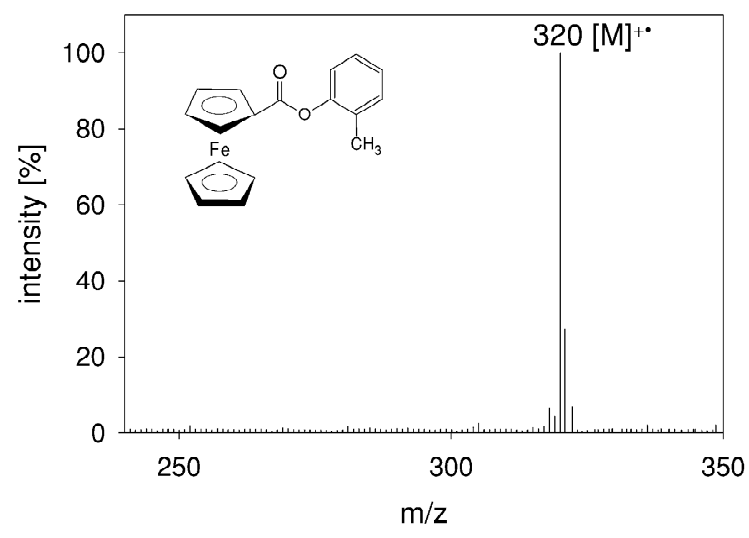

Fig. 4. $\mathrm{APCI}(+)$ mass spectrum of 2-cresol FCE. Inserted: Chemical structure of the analyte.

ent alkylphenyl ferrocenecarboxylic acid esters of chain lengths $\mathrm{C} 0$ to $\mathrm{C} 6$ and $\mathrm{C} 8$ and $\mathrm{C} 9$ in acetonitrile was analyzed utilizing different gradients. The optimum gradient was found to be gradient B. Fig. 5 shows the resulting total ion current (TIC) chromatogram. Although the mixture contained the unpolar derivatives of 4- $n$-octylphenol and 4- $n$-nonylphenol and the gradient had to be kept at a high acetonitrile concentration for a longer time to elute these two analytes, the separation in Fig. 5 was accomplished in less than $1.5 \mathrm{~min}$. No baseline separation could be achieved but the separation is sufficient for quantification even when using the extracted mass traces from the TIC. Using the selected ion monitoring (SIM) traces provides for additional sensitivity with respect to qualitative or quantitative determinations.

Since the first analyte phenyl FCE elutes at $19 \mathrm{~s}$, there is still significant peak capacity available in the chromatogram for more polar interferences that might elute earlier than the phenyl FCE. In general, the derivatives are eluting in the sequence of increas-
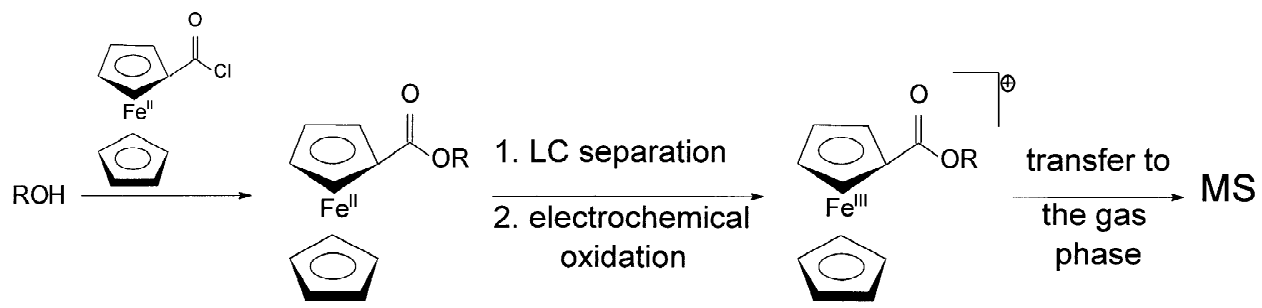

Fig. 3. Derivatization of alcohols and alkylphenols to the corresponding ferrocenecarboxylic acid esters, LC separation and on-line electrochemical oxidation to the ferrocinium radical cations detectable by APCI-MS. 


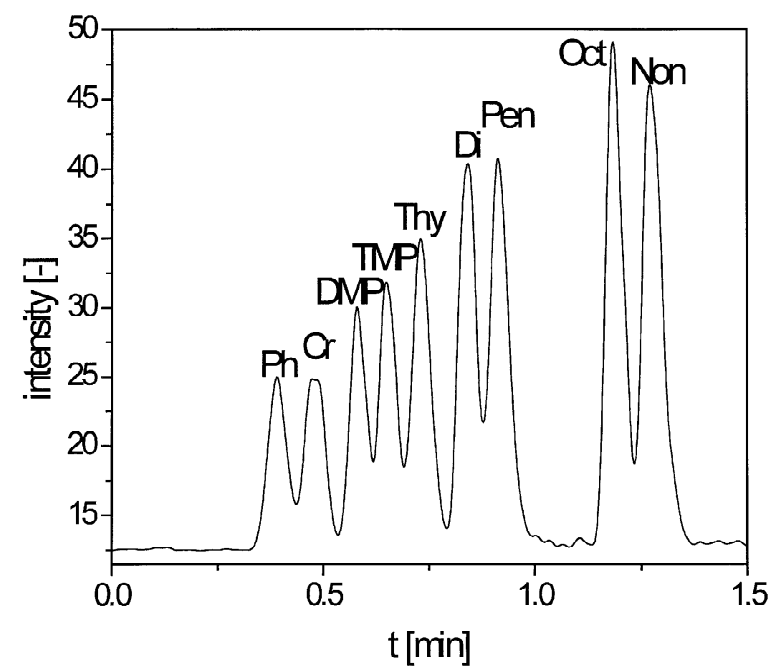

Fig. 5. Fast LC-electrochemistry-APCI(+)-MS TIC chromatogram, recorded in the SIM mode $(\mathrm{m} / \mathrm{z}=306.0,320.1,334.1$, 348.1, 362.1, 376.1, 390.1, 418.2, 432.2); $\mathrm{Ph}=$ phenyl FCE, $\mathrm{Cr}=2$ cresol FCE, DMP=2,5-dimethylphenyl FCE, TMP=2,3,6-trimethylphenyl FCE, Thy=thymol FCE, Di=2,6-diisopropylphenyl FCE, Pen $=4-n$-pentylphenyl FCE, Oct $=4-n$-octylphenyl FCE, Non=4-n-nonylphenyl FCE.

ing alkyl side chain length. As it can be expected because of its more spherical molecular shape, 2,6diisopropylphenyl FCE elutes before the 4pentylphenol derivative.

\subsection{Fast LC-electrochemistry-MS of alcohol derivatives}

Since it was possible to employ fast LC-electrochemistry-MS utilizing guard columns for the determination of several alkylphenol derivatives, the same approach was used for the determination of different alcohol derivatives. A $10 \mu M$ solution of the derivatives of methanol, ethanol, 1-propanol, 2butanol, 1- $n$-pentanol and 1- $n$-octanol in acetonitrile was separated using gradient $\mathrm{C}$ in $1 \mathrm{~min}$ (Fig. 6). As in the previous figure, the first analyte methyl FCE is already retained significantly under these conditions. Again, no baseline separation was accomplished. Although the separation efficiency was not as good as in the chromatogram of Fig. 2, it is still sufficient for quantification in TIC or SIM traces, as is proven by the quantitative data in Table 2 .

Single SIM traces were integrated for the quantita-

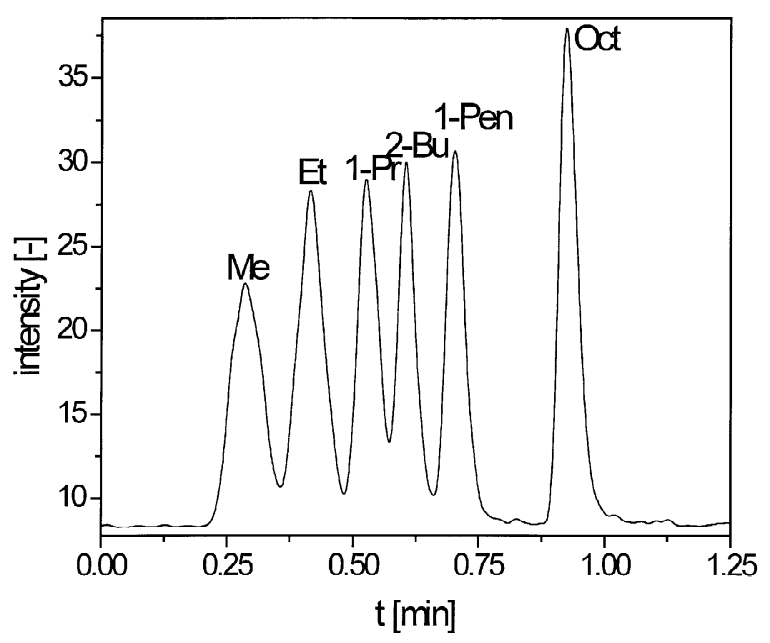

Fig. 6. Fast LC-electrochemistry-APCI(+)-MS TIC chromatogram, recorded in the SIM mode $(m / z=244.0,258.0,272.1$, 286.1, 300.1, 342.1); Me=methyl FCE, Et=ethyl FCE, 1-Pr=1propyl FCE, 2-Bu=2-butyl FCE, 1 -Pen $=1-n$-pentyl FCE, Oct $=1$ $n$-octyl FCE.

tive data in Table 2. The limit of detection (LOD) was determined as a signal-to-noise ratio of 3 and the limit of quantification (LOQ, not provided in the table) as a signal-to-noise ratio of 10 . The relative standard deviation (RSD) for three subsequent analyses was determined at a concentration of $0.5 \mu M$.

Table 2

Analytical figures of merit for selected FCE derivatives (LOD= limit of detection, $\mathrm{LOQ}=$ limit of quantification, $\mathrm{RSD}=$ relative standard deviation), detection in the SIM mode

\begin{tabular}{lcc}
\hline Analyte & $\begin{array}{l}\text { LOD } \\
(\mathrm{n} M)\end{array}$ & $\begin{array}{l}\text { RSD }(\%) \\
(n=3 ; c=0.5 \mu M)\end{array}$ \\
\hline Methyl FCE & 140 & 6 \\
Ethyl FCE & 130 & 8 \\
1-Propyl FCE & 140 & 4 \\
2-Butyl FCE & 70 & 10 \\
1-Pentyl FCE & 60 & 9 \\
1-Octyl FCE & 80 & 7 \\
Phenyl FCE & 80 & 5 \\
2-Cresol FCE & 130 & 4 \\
2,5-Dimethylphenyl FCE & 140 & 4 \\
2,3,6-Trimethyphenyl FCE & 190 & 7 \\
Thymol FCE & 140 & 6 \\
4-Pentylphenyl FCE & 120 & 5 \\
2,6-Diisopropylphenyl FCE & 170 & 5 \\
4- $n$-Octylphenyl FCE & 60 & 7 \\
4- $n$-Nonylphenyl FCE & 80 & 8 \\
\hline
\end{tabular}


As Table 2 shows, the limits of detection were in the range of 60 to $190 \mathrm{nM}$. This is slightly higher than the LODs that were reported for LC-electrochemistry-APCI(+)-MS with conventional LC separations [6]. This may be due to the higher detector sampling rate required by fast LC. A linear concentration range of two decades starting at the LOQ was observed for all derivatives. The relative standard deviation for three runs was between 4 and $10 \%$.

\subsection{Rapid LC-electrochemistry-MS using graphite filter elements as stationary phases}

During work with the conventional LC-electrochemistry-MS system $(0.5 \mathrm{ml}$ volume mixing chamber, standard analytical columns of $150 \mathrm{~mm}$ length and graphite in-line filter; see Ref. [6]), and when using an on-line UV-Vis detector between the column and the electrochemical cell, a considerable time delay between UV-Vis and mass spectrometric signals was noticed. This was not due to the additional void volume of the electrochemical cell. Moreover, the time delay increased from the derivatives of the short chain length alcohols to those of the larger phenols like $n$-nonylphenol. Further investigations have been undertaken to explain this effect and to use it in a LC-electrochemistry-MS system without a dedicated chromatographic column.

To investigate if the observed separation is due to retention on the glassy carbon material of the electrochemical cell or on the graphite material of the in-line filter, the graphite filter material was exchanged by PEEK material. Under these conditions, no additional retention was observed for any of the derivatives, indicating that the graphite material of the in-line filter was responsible for the effect.

Different lots of graphite filter were used to test the reproducibility of the separation. Due to asymmetric peak shape, the retention times of the of very early eluting substances varied by up to $15 \%$ between filter elements and up to $6 \%$ for different injections on the same filter element. The retention times of several well retained analytes varied only slightly between the filter elements (less than 3\%) and for different injections on the same filter element (less than 1\%), thus encouraging experiments with respect to their use as stationary phases. Although the material is characterized by a thickness of only $1 \mathrm{~mm}$, experiments to use the filter instead of a chromatographic column were carried out in the following.

The SIM traces of one of the resulting electrochemistry-APCI(+)-MS chromatograms using a graphite in-line filter and gradient $\mathrm{E}$ are shown in Fig. 7. Although the peak shape is not even close to optimal, a separation of some of the FCEs can be accomplished. The FCEs of the alcohols from methanol to 2-propanol elute first, with no separation and are followed by the partially separated FCEs of $1-n$-butanol and 1-n-pentanol. Interestingly, the rather large but spherical 2,6-diisopropylphenyl FCE elutes next, a short time before the smaller 4-cresol derivative. As expected, the long chain alkylphenol FCEs of $n$-octyl- and $n$-nonylphenol elute last. This may be explained by the interaction of the aromatic structures with the planar graphite material. This interaction is reduced by bulky substituents at the aromatic ring and results in an elution of the larger 2,6-diisopropylphenol derivative in front of several of the smaller derivatives.

\section{Conclusion}

It was demonstrated that fast LC separations over conventional guard columns can be used with the new technique of LC-electrochemistry-APCI $(+)$ MS. Although no baseline separation was achieved for different mixtures of FCE standards, efficient separation sufficient for identification and quantification could be accomplished in less than $1.5 \mathrm{~min}$; this is an improvement of more than factor 10 , as compared to the use of standard length analytical columns.

In addition it was shown that retention occurs of several alcohol and phenol derivatives on the graphite filter element employed in the electrochemistry set-up. Based on this finding, a separation without chromatographic column and with mass spectrometric detection of different standards was accomplished using the graphite filter as stationary phase. Although the separation over dedicated columns is superior, the filter approach is characterized by a very simple experimental setup. In combination 


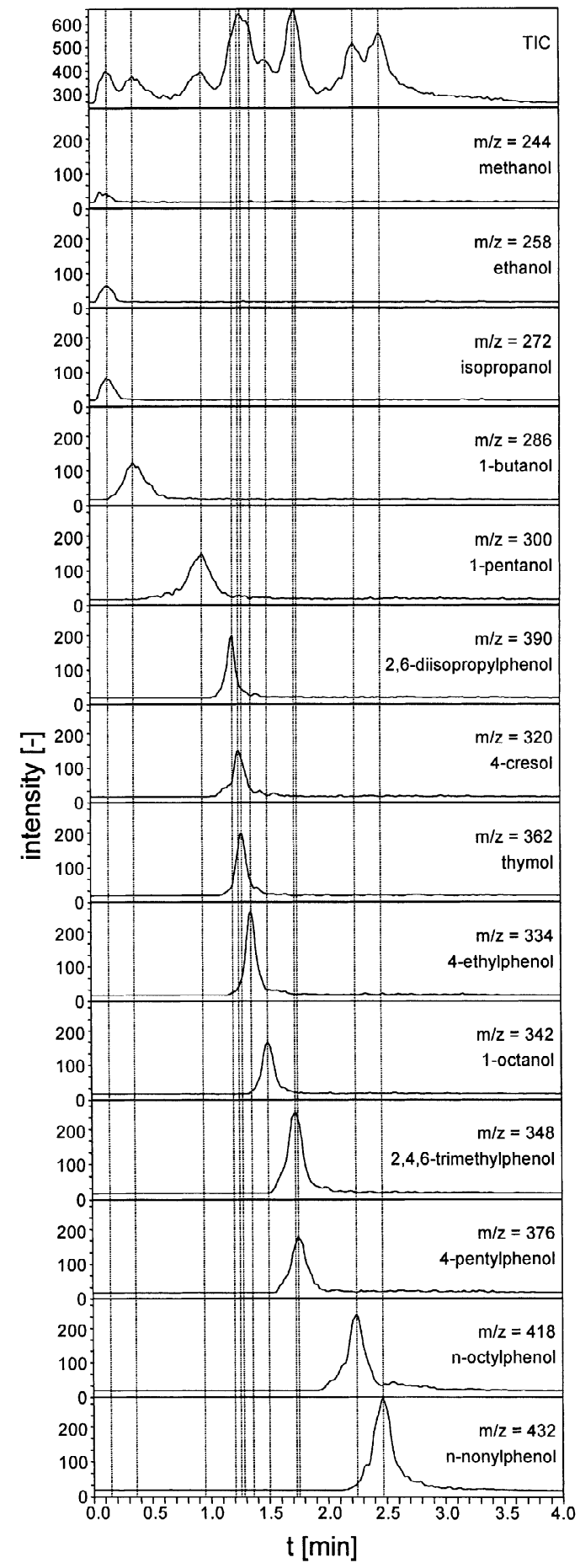

Fig. 7. Graphite in-line filter based separation of the FCE derivatives of selected alcohols and phenols employing LCelectrochemistry-APCI(+)-MS, recorded in the SIM mode. with mass spectrometric detection, the separation achieved here is sufficient for many analytical problems.

\section{Acknowledgements}

Several alkylphenyl FCE standards were kindly provided by F. Wasinski, J. Rolfes and J.T. Andersson from the University of Münster (Münster, Germany). Financial support by the Fonds der Chemischen Industrie (Frankfurt a. M., Germany) is gratefully acknowledged.

\section{References}

[1] W.M.A. Niessen, J. Chromatogr. A 856 (1999) 177.

[2] R.B. Cole (Ed.), Electrospray Ionization Mass Spectrometry, Wiley, New York, 1997.

[3] G.J. Van Berkel, K.G. Asano, Anal. Chem. 66 (1994) 2096.

[4] K.J. Volk, R.A. Yost, A. Brajter-Toth, Anal. Chem. 64 (1992) $21 \mathrm{~A}$.

[5] G. Diehl, U. Karst, Anal. Bioanal. Chem. 373 (2002) 390.

[6] G. Diehl, A. Liesener, U. Karst, Analyst 126 (2001) 288.

[7] L.L. Lloyd, F.P. Warner, J. Chromatogr. 512 (1990) 365.

[8] N.B. Afeyan, N.F. Gordon, I. Mazsaroff, L. Varady, S.P. Fulton, Y.B. Yang, F.E. Regnier, J. Chromatogr. 519 (1990) 1.

[9] K.K. Unger, G. Jilge, J.N. Kinkel, M.T.W. Hearn, J. Chromatogr. 359 (1986) 61.

[10] K. Kalghati, Cs. Horváth, J. Chromatogr. 398 (1987) 335.

[11] H. Chen, Cs. Horváth, J. Chromatogr. A 705 (1995) 3.

[12] M. Hanson, K.K. Unger, LC.GC 9 (1996) 741.

[13] A.C. Hogenboom, J. Slobodnik, J.J. Vreuls, J.A. Rontree, B.L.M. van Baar, W.M.A. Niessen, U.A.Th. Brinkman, Chromatographia 42 (2001) 506.

[14] A.C. Hogenboom, U.K. Malmqvist, K. Nolkrantz, J.J. Vreuls, U.A.Th. Brinkman, J. Chromatogr. A 759 (1997) 55.

[15] A.C. Hogenboom, W.M.A. Niessen, U.A.Th. Brinkman, J. Chromatogr. A 794 (1998) 201.

[16] F. Zhou, G.J. Van Berkel, Anal. Chem. 67 (1995) 3643.

[17] J. Rolfes, J.T. Andersson, Anal. Commun. 33 (1996) 429.

[18] J. Rolfes, J.T. Andersson, Anal. Chem. 73 (1996) 3073. 